\title{
The double-edged sword of CRISPR-Cas systems
}

\author{
Manuela Villion ${ }^{1}$, Sylvain Moineau ${ }^{1}$ \\ 'Département de Biochimie, de Microbiologie et de Bio-informatique, Faculté des Sciences et de Génie, Groupe de Recherche \\ en Écologie Buccale, Faculté de Médecine Dentaire, Félix d'Hérelle Reference Center for Bacterial Viruses, Université Laval, \\ Québec City, Quebec G1V 0A6, Canada
}

Cell Research (2013) 23:15-17. doi:10.1038/cr.2012.124; published online 4 September 2012

\section{A recent paper gives the details on how specific small RNAs can program a protein to cleave an undesired piece of DNA and to provide immunity to a microbial cell.}

The number of microbes on Earth has been estimated at $10^{30}[1]$ and their viruses are believed to outnumber them by at least 10 -fold. Consequently, viruses of microbes are considered the most abundant and diversified biological entities on our planet [2].

To cope with this never-ending threat, microorganisms have developed a wide range of defense mechanisms [3]. Among them, CRISPR-Cas system is the new kid on the block as its silencing role was reported only five years ago [4]. An outburst of articles, meetings, and reviews has since followed, arguably making it one of the hottest topics in microbiology.

CRISPR (clustered regulatory interspaced short palindromic repeats) loci are found in approximately $45 \%$ of sequenced bacterial genomes as well as $90 \%$ of archaeal ones and one genome can contain multiple CRISPR loci. Variable short regions, called spacers, separate each of the short repeats. The spacers are mainly homologous to viral or plasmid sequences. CRISPR-associated (cas) genes are often located adjacent to

Correspondence: Sylvain Moineau Tel: +1-418-656-3712; Fax: +1-418-656-2861

E-mail: Sylvain.Moineau@bcm.ulaval.ca the CRISPR locus [5]. The diversity and specificity of the cas operons has led to the identification of signature cas genes and to a polythetic classification scheme for CRISPR-Cas systems (types I to III, with several subtypes) [6].

Notwithstanding their particularities, CRISPR-Cas systems operate through three general steps to provide immunity. In the adaptation stage, some cells will respond to the invasion of a phage or a plasmid by adding a new repeat-spacer unit into the CRISPR array, mostly polarized at the $5^{\prime}$ end. Strikingly, the spacer sequence comes from the invading nucleic acid while the newly added repeat derives from another repeat of the array. The mechanistic details on how this adaptation/immunization occurs are still unknown but some Cas proteins are involved. The unique spacer content is now considered a sign of past challenges and can serve as a marker for strain typing [5].

In the second step, small non-coding CRISPR RNAs (crRNAs) are generated. A long precursor CRISPR RNA is first produced from an AT-rich leader/promoter region, which is then processed within the repeats and mature into crRNAs. Several Cas proteins participate into the biogenesis of crRNAs. Finally, in the interference stage, the crRNACas protein complex will bind to the invading nucleic acid target and cleave it, providing a defense system to the host microbe [5]. Therefore, CRISPR-
Cas systems are RNA-based adaptive microbial immune systems that target nucleic acid intruders.

Type II systems have been heavily studied partly because they offer practical applications in the dairy industry to generate phage-resistant Streptococcus thermophilus strains [4] and partly because the main functional steps have been experimentally confirmed. The repeats are generally $36 \mathrm{bp}$ long while the spacers are $30 \mathrm{bp}$. In addition to its content and architecture, type II systems also differ from other types in the biogenesis of crRNAs. Indeed, another set of small non-coding RNAs ( $\sim 100$ nucleotides (nt)) that are partially complementary to the type II CRISPR repeat, are produced from a region outside but close to the CRISPR locus. These small RNAs are called tracrRNA, for trans-activating CRISPR RNA. These tracrRNAs hybridize to the repeats within the long precursor CRISPR RNA and the RNA duplexes are processed by the non-CRISPR RNase III to generate mature crRNAs ( $\sim 42 \mathrm{nt})$, with roughly half matching the spacer and half matching the repeat [7].

Cas9, a large type-II signature protein, was shown to be the only Cas protein involved in this biogenesis process [7]. Cas9 was also shown to be somehow essential for the cleavage of phage or plasmid dsDNA target during the interference stage in $S$. thermophilus $[8,9]$. 
The recent stimulating paper by Jinek et al. [10] shed light on the mechanistic role of Cas 9 in the interference stage of the CRISPR-Cas type II systems. Cas9 contains at least two nuclease domains, a RuvC-like domain near the amino terminus and the $\mathrm{HNH}$ (or McrA-like) nuclease domain in the middle of the protein. Using in vitro assays and a purified Cas9 from Streptococcus pyogenes, the authors showed that each domain is involved in the cleavage of one strand of the dsDNA target. The RuvC-like domain cleaves the noncomplementary strand while the HNH domain acts on the complementary strand. Evidence was also provided that Cas9 is a multiple-turnover enzyme that can cleave both linearized and supercoiled plasmids. Overall, the cleavage rate of Cas9 was comparable to the ones observed for restriction endonucleases.

Remarkably, two RNA molecules, tracrRNA and crRNA, the latter having a sequence complementary to the DNA target, are absolutely required for target DNA binding and cleavage by Cas 9 endonuclease. Therefore, Cas 9 and the small non-coding tracrRNA (both specific to type II systems) are involved in the maturation of crRNAs (biogenesis of crRNA stage) as well as in the cleavage of the target dsDNA (interference stage). Moreover, through in vitro studies with Cas 9 orthologs, the authors also showed that target cleavage was microbial species specific, suggesting a co-evolution of Cas 9 , tracrRNA, and the repeats.

Using a plasmid or a short linear dsDNA as an in vitro target, the authors determined that the cleavage produced blunt ends, three base pairs upstream of a short motif called PAM (protospacer adjacent motif). A protospacer is defined as the nucleotide sequence found in the invading nucleic acid that is matching the spacer in the CRISPR array. The PAM is flanking the protospacer and is thus found in the invading sequence, thereby playing a role in distinguishing self (spacer, host) from non-self (pro- tospacer, foreign) [11]. Interestingly, this cleavage site perfectly matched the one observed in vivo in phage-infected or plasmid-containing $S$. thermophilus strains $[8,12]$.

Of note, using a short linear substrate, Jinek et al. [10] revealed that both strands are cleaved in different ways. While the complementary strand to crRNA is cleaved precisely three bases upstream of the PAM, the noncomplementary strand could be cleaved elsewhere and needs further $3^{\prime}-5^{\prime}$ trimming to reach the same position.

Using binding assays, Jinek et al. [10] also suggested that Cas9 recognized PAM sequences as a prerequisite for target DNA binding and possibly strand separation to allow R-loop formation (a structure in which crRNA molecule would hybridize with one strand of a dsDNA target, leaving the other strand unpaired). Indeed, binding affinity was enhanced with a perfect matched PAM.

Taken altogether, Cas9-tracrRNAcrRNA complexes would include basepairing between $22 \mathrm{nt}$ of the mature crRNA with the tracrRNA through matching repeat portion, leaving $20 \mathrm{nt}$ of the crRNA available for target DNA binding. In fact, it was shown that only 13 bp between the crRNA and the protospacer were required for efficient target cleavage. The $13 \mathrm{bp}$ adjacent to the PAM, could be seen as a seed region defining requirements for Cas9 binding to the target. As for the rest of the tracrRNA, it would be available to interact with Cas9 or to form other RNA structure or bind to other partners. Structural work on this complex should help refine the model.

Although already outstanding in bridging gaps in our understanding of CRISPR-Cas systems, this fascinating story does not end here. The authors investigated the possibility of using this dual-RNA system to program Cas9 to specifically cleave any desired DNA molecules. Minimal requirements to have an efficient single chimeric RNA molecule mimicking the dual RNA structure were defined and led to sitespecific DNA cleavage by Cas9. In fact, several different chimeric guide RNAs were engineered and used to cleave a plasmid containing the specific target and a PAM. These findings coupled to the previous observations that CRISPRCas systems can be functionally transferred from one organism to another [9] open up exciting possibilities for gene targeting and genome editing of microbes and even higher organisms [13].

The work of Jinek et al. [10] represents another exciting chapter of the ever-growing story of CRISPR-Cas systems. Knowledge gaps are being filled at a stunning speed, its mode of action is becoming clearer, and novel stimulating biotechnological applications keep emerging. The next major challenge certainly lies in better understanding the adaptation stage, which has been difficult to study due to, among others, the low frequency of cells acquiring novel repeat-spacer units. The diversity of the CRISPR-Cas systems also undoubtedly reserve additional surprises in the forthcoming years and should keep interested readers looking forward to the next installment.

\section{References}

1 Whitman WB, Coleman DC, Wiebe WJ. Prokaryotes: The unseen majority. Proc Natl Acad Sci USA 1998; 95:6578-6583.

2 Edwards RA, Rohwer F. Viral metagenomics. Nat Rev Microbiol 2005; 3:504-510.

3 Labrie SJ, Samson J, Moineau S. Bacteriophage resistance mechanisms. Nat Rev Microbiol 2010; 8:317-327.

4 Barrangou R, Fremaux C, Deveau H, et al. CRISPR provides acquired resistance against viruses in prokaryotes. Science 2007; 315:1709-1712.

5 Wiedenheft B, Sternberg SH, Doudna JA. RNA-guided genetic silencing systems in bacteria and archaea. Nature 2012; 482:331-338.

6 Makarova KS, Haft DH, Barrangou R, et al. Evolution and classification of the 
CRISPR-Cas systems. Nat Rev Microbiol 2011; 9:467-477.

7 Deltcheva E, Chylinski K, Sharma CM, et al. CRISPR RNA maturation by trans-encoded small RNA and host factor RNase III. Nature 2011; 471:602607.

8 Garneau JE, Dupuis ME, Villion M, et al. The CRISPR/Cas bacterial immune system cleaves bacteriophage and plasmid DNA. Nature 2010; 468:67-71.

9 Sapranauskas R, Gasiunas G, Fremaux
C, Barrangou R, Horvath P, Siksnys V. The Streptococcus thermophilus CRIS$\mathrm{PR} /$ Cas system provides immunity in Escherichia coli. Nucleic Acids Res 2011; 39:9275-9282.

10 Jinek M, Chylinski K, Fonfare I, Hauer M, Doudna JA, Charpentier E. A programmable dual-RNA-guided DNA endonuclease in adaptive bacterial immunity. Science 2012; 337:816-821.

11 Mojica FJ, Díez-Villaseñor C, GarcíaMartínez J, Almendros C. Short motif sequences determine the targets of the prokaryotic CRISPR defence system. Microbiology 2009; 155:733-740.

12 Magadán AH, Dupuis ME, Villion M, Moineau S. Cleavage of phage DNA by the Streptococcus thermophilus CRISPR3-Cas system. PLoS One 2012; 7:e40913.

13 Perez-Pinera P, Ousterout DG, Gersbach CA. Advances in targeted genome editing. Curr Opin Chem Biol 2012; 16:268-277. 